\title{
Editorial \\ Corporate Social Responsibility: Organizational Strategy for Sustainable Growth
}

\author{
Shufeng(Simon) Xiao ${ }^{1}$ (D) and Byung Il Park ${ }^{2, *}$ \\ 1 Division of Business Administration, Sookmyung Women's University, Seoul 04310, Korea; \\ bizsxiao@sookmyung.ac.kr \\ 2 Hankuk University of Foreign Studies, College of Business, Seoul 130791, Korea \\ * Correspondence: leedspark@hufs.ac.kr; Tel.: +82-221733193
}

check for updates

Citation: Xiao, S.; Park, B.I. Corporate Social Responsibility: Organizational Strategy for Sustainable Growth. Sustainability 2021, 13, 13589. https://doi.org/ $10.3390 /$ su132413589

Received: 3 December 2021 Accepted: 4 December 2021 Published: 9 December 2021

Publisher's Note: MDPI stays neutral with regard to jurisdictional claims in published maps and institutional affiliations.

Copyright: (c) 2021 by the authors. Licensee MDPI, Basel, Switzerland. This article is an open access article distributed under the terms and conditions of the Creative Commons Attribution (CC BY) license (https:/ / creativecommons.org/licenses/by/ $4.0 /)$.
Welcome to the special issue of Sustainability on "corporate social responsibility: organizational strategy for sustainable growth." We are delighted to publicize that ten research studies have been selected for publication in the special issue after our in-depth tripleblind review process. We trust that all studies discussed the important discourse on the topic that this special issue pursues. Prior to explaining what the ten studies addressed, a special-issue background is given below:

\section{Special Issue Background}

Cateora and Graham [1] introduced an interesting management and business case in their book, in which one of the main characters in the case argues the following (p. 634):

“... Things are just done different here. You follow that policy and I guarantee that you'll have fewer sales because our competitors from Germany, Italy, and Japan will pay (bribe). Look, Bill, everybody does it here; it's a way of life ... the Latinos even pay mordidas (i.e., bribe) to other Latinos; it's a fact of life-I think that the circumstances that exist in a country justify and dictate the behavior. Remember man, 'When in Rome, do as the Romans do'".

The short quote above questions whether unethical behavior (corporate irresponsible activities) undertaken by international firms in developing countries is really entirely unethical or simply unavoidable grease, which is necessary in facilitating short-term growth in overseas markets. Both strategic-management and international-business scholars have attempted to examine the outcomes of corporate social responsibility primarily in the organizational strategic aspect. Due to this, they are turning a blind eye to firms' unethical attitudes and are pretending not to notice that it (i.e., unethical behavior) is happening. Current discussions dealing with corporate social responsibility (CSR) have concentrated on solving queries, such as what promotes a firm's citizenship, under which environments firms conduct ethical management, what the primary determinants affecting a certain CSR (e.g., philanthropic CSR) are, and how it strategically enhances corporate performance, but such discussions tend to overlook its effect on long-term sustainable growth.

Thus, we do not yet know enough about CSR from a long-term perspective and its relationship with long-term sustainable growth. We should also acknowledge that a firm's unethical management is a primary cause and a result of poverty in our economy, which means that a firm's irresponsible activities (as an antonym of CSR) cannot be justified by any reason. This also represents the importance of research exploring CSR.

To dig further into CSR per se, it can be generally defined as voluntary actions by firms that appear to further some social good, where the activity level is "above and beyond" what is required by law, though current CSR frameworks are various [2]. As discussed above, there is no doubt that being socially responsible is crucial in itself, and organizations must make a sensible decision about the level to which they leverage their CSR activities. Then, they should translate doing good into strategic benefits [3-5]. Through the strategies based on the CSR, firms often move to a situation where they use organizational core 
resources to improve their competitiveness and performance. Considering the treasurable value of CSR, the management research handling the topic is in the limelight and has shown that firms undertaking stronger CSR are usually eligible to attract consumers (in marketing), they commonly fulfill the expectations of investors (in finance), they satisfy internal employees (in management), and they successfully compete against local and global competitors (in strategic international business).

First, as we have sufficiently accumulated CSR research in the different management areas, we believe that this is the time to synthesize diverse research fragments on CSR. Second, we should combine it with an additional unique agenda (e.g., human rights, win-win partnership, and official development assistance), particularly in the sustainability domain, and then compile all theoretical and empirical pieces for organizational sustainability. Thus, by inviting submissions from researchers who study various theoretical perspectives, who adopt various approaches, and who examine at multiple levels of analysis, while including qualitative and quantitative experiments, literature reviews, and meta-analyses, this special issue draws a large picture. In this vein, we are strongly convinced that the studies accepted by this special issue significantly contribute to current relevant debates by filling many extant research gaps. Please see the details below:

\section{The Ten Studies Included in This Special Issue}

The first article written by Huifang Liu and Jin-Sup Jung identifies key characteristics of corporate social responsibility (CSR) influencing a firm's CSR authenticity. By using regression analyses, the authors uncovered that all three attributes of CSR (i.e., CSR fit, CSR sustainability, and CSR effect) have meaningful relationships with CSR authenticity. However, compared with other attributes, CSR sustainability had relatively marginal associations with CSR authenticity in the Korean sample, whereas the CSR fit did not significantly determine CSR authenticity in the Chinese one. The authors determined the fundamental reason for the different results from different consumer characteristics between the two countries. By contrast, they also detected that the digital transformation of the Fourth Industrial Revolution has strong partial mediating effects between CSR attributes and CSR authenticity. According to them, this result denotes that digital transformation may be an important pathway to obtain CSR authenticity and also indicates that the mediating effect functions as a conduit to a firm's competitiveness.

The second article authored by Arnold Bernaciak, Małgorzata Halaburda, and Anna Bernaciak sought to answer what motivates, retards, and determines a firm's CSR in the context of the Polish construction sector. In addition, this study also attempted to explore the effects of firm size (i.e., large, medium, and small) on the issue illustrated above. The authors explained the importance of their examination by determining that the construction industry has a considerable effect on the environment and people, uses an enormous volume of natural resources, generates polluted materials, and mass produces large amounts of waste. Moreover, CSR is a vital agenda in that construction sites are a place of danger and accidents. Based on the triangulation method, the study discovered the presence of large variations with respect to motives, barriers, and expected benefits out of the execution of CSR principles. Their findings also revealed that the practice of CSR principles is often affected by a firm's various considerations and situations. That is, it may be difficult to assert that a certain factor clearly determines the CSR activities. The results achieved may indicate a useful implication for decision-makers at the management level and for legislation, thus regulating better conditions for the development of CSR in the sector.

Kum-Sik Oh, Juyeon Rachel Han, and So Ra Park, who authored the third article, attempted to examine the relationships among the employees' perception of CSR, their intrinsic motivations, and their organizational commitment in the hotel industry. In addition, they also explored (1) the mediating effect of intrinsic motivation on the association between the employees' perception of customer- and employee-related CSR and organizational commitment, and they tested (2) the moderating role of job level on the relationship 
between CSR perceptions and intrinsic motivation. According to the results obtained from quantitative analyses, they uncovered that (1) both types of CSR perceptions are crucial in fostering intrinsic motivation and organizational commitment; (2) intrinsic motivation improves organizational commitment; and (3) the link between employee CSR perceptions and intrinsic motivation is positively moderated by job level. Another interesting result detected by them is that the level of intrinsic motivation will be considerably different between managerial and non-managerial employees in the case where customer-related CSR or employee-related CSR is high. Such findings may be useful for human-resource departments, in which CSR practices can be a means to enhance employee intrinsic motivation and organizational commitment.

The main objective of the fourth study by Mengmeng Wang and Xue Fan was to analyze the fitness between live streaming e-commerce and green agrifood. To answer the question of how livestreaming can contribute to the sustainability of green agri-food entrepreneurial firms, the theory of a task-technology fit was employed. The authors collected the data through a web-based questionnaire and obtained them from a sample of 574 green agri-food entrepreneurial firms. Then, they ran a structural-equation-modeling (SEM) analysis and determined a positive effect of the locality and eco-friendliness of green agri-food, the responsiveness, interactivity, and entertainment of livestreaming e-commerce on the fit of green agri-food livestreaming e-commerce. Furthermore, their results demonstrated that the fit of green agri-food livestreaming has a positive effect on firm performance and the intention to adopt livestreaming e-commerce. The study also shows the moderating effect of perceived CSR on the relationship between the fit of livestreaming of green agri-food and the intention to adopt livestreaming e-commerce. Their findings are valuable because they provide useful insights into the successful adoption of livestreaming e-commerce.

The fifth study written by Xiaoyan Pan, Kum-Sik Oh, and Mengmeng Wang started developing the idea that strategic orientation represents an essential antecedent condition for new product development (NPD) performance, which can be a vehicle for firms to improve their competitive advantage and enlarge sustainable growth. Under this premise, the study investigated how strategic orientation (i.e., technology orientation and customer orientation) promotes a firm's digital capabilities and NPD performance in the context of digital transformation. Using a resource-based view and its extended dynamic capabilities as an overarching theoretical lens, it tried to solve the doubt. Their analyses from SEM shed light on the roles of technology orientation and customer orientation and revealed that both factors can be instrumental in driving a firm's digital capabilities (if we compare the influential powers of the two factors, technology orientation plays a more pivotal role than customer orientation in contributing to NPD performance). Moreover, their experiment also confirmed the moderating effect of CSR on the relationship between strategic orientation and NPD performance. It provides an important insight into non-market mechanisms and emphasizes that firms can compensate for their strategically-oriented practices through CSR in the NPD process.

The sixth article by two authors (i.e., Boine Kim and Byoung-Goo Kim) noted that extant empirics investigating the outcomes of the venture firms' CSR initiatives are scant. In this vein, it aimed to analyze the factors influencing their performance and indicated that both financial and non-financial firm growth is an end product of CSR activities. From statistical analyses on data collected from Korean venture firms, the message given by the study is clear. That is, according to the results yielded by step-wise regression analyses, it determined that the number of CSR types (e.g., donation, talent donation, community service, creating shared value, sponsorship, etc.) in which firms participate is a crucial determinant promoting organizational development. From the results, the authors argued that firms do not only need to conduct CSR but also should sincerely try to diversify ethical endeavors.

The seventh study was another empirical study conducted by Bu-Kyung Choi, JiYoung Ahn, and Myeong-Cheol Choi. They examined the economic effect of CSR initiatives on corporate innovation, and Korean firms were employed for the investigation. The main 
objective of the study was that the authors attempted to explain how a CEO compensation system can affect the CSR-innovation relationship. To solve their curiosity, they developed an integrated model exploring the effect of CSR on innovation activities through analyzing various factors comprising CEO compensation schemes (e.g., structure, type, mix, and distribution). Using longitudinal data, the empirical study yielded the following results. First, CSR can ignite innovation activities. Second, in the case where the proportion of performance-based pay to total compensation is great, the positive connection between CSR and innovation activities is strengthened. Third, the evidence on the potential alignment between CSR and the intensity of stock-based pay is unclear. Fourth, the wage gap between the $\mathrm{CEO}$ and the top management team undermines the positive effect of CSR on innovation activities. Based on the empirical outcomes, this study provides important insights into the CSR-innovation relationship.

Another experiment undertaken by Xinyuan Wang, Zhenyang Zhang, and Dongphil Chun scrutinized the potential connection among internal control effectiveness, CSR, and technological innovation. The eighth study employed the data of Chinese A-sharelisted firms between 2014 and 2019 as its sample. The important discoveries achieved by the study are threefold. First, the internal control effectiveness was significantly and positively associated with technological innovation. Second, the betterment of internal control effectiveness had a significant and positive influence on CSR performance. Third, CSR plays a partial mediating role in linking internal control effectiveness and technological innovation. These findings indicated that internal control effectiveness can be a vehicle to promote technological innovation through the transmission mechanism of CSR.

The aim of the ninth article, which was written by Mengmeng Wang and Wenjie Yang, was to depict a large picture that may help better understand what determines rural consumers' positive attitudes on e-commerce and how it can improve their willingness to adopt it. In contrast with other studies, which are primarily based on quantitative techniques, this study, as a first step, used a qualitative interview-based experiment of 104 rural consumers to test the framework designed. According to the authors' arguments, these practices are suitable in identifying new problems or challenges often encountered by rural consumers in impoverished areas after several years of experience in e-commerce. Posterior to the qualitative attempt, this study also conducted additional quantitative tests to further examine the effects of service quality and cultural context. A total of 434 rural consumers in relatively underdeveloped areas of China are participated in the survey. The SEM results of this study exhibited a positive association between logistics and training service quality, subjective norms, self-efficiency sense, and the rural consumers' attitudes toward e-commerce platforms, respectively. The authors insisted that the confirmed relationship above indicated the consumers' positive intent to engage in word-of-mouth e-commerce promotion. Moreover, the study also revealed the positive moderating effects of CSR on logistics and training services, subjective norms, and attitudes toward the use of e-commerce platforms. The discoveries from these two studies provided practical implications and significantly contributed to both CSR and e-commerce literatures.

The final study by Márcia Machado and Tereza Carvalho was based on exploratory research navigating the academic databases of Web of Science, IEEE, Scopus, and Google Scholar to identify works that address sustainability and other relevant concepts. This process was conducted to examine the association between control objectives for information and related technologies (COBIT) maturity models adopted by information technology (IT) firms and the sustainability indicators. In other words, this study sought to develop a series of sustainable indicators, which can be adopted by small- and medium-sized software firms, and investigated the relationship between those indicators and the COBIT maturity model. The authors indicated that this set of indicators can be the essential foundations for CSR and environmental, social, and corporate governance (ESG). In particular, in the perspectives of ESG, the authors uncovered that the environmental and social indicators have a closer connection with the COBIT model than others (e.g., economic indicators). Moreover, the authors also highlighted that governance often functions as a strategic mechanism 
that controls the balance of activities and facilitates the adequate use of organizational resources. Thus, the mechanism comprising governance has a propensity to improve corporate performance and efficiency.

The guest editors noted that the authors, who submitted to the special issue, commonly provided the criticism that although CSR has been considered as a critical issue for marketers, managers, and top management the concept has been only recently attracting scholarly attention. Due to the problem, many issues on CSR have been veiled, and many extant studies have not been adequately rooted in theoretical basis. Although we acknowledged some of the exceptions, previous studies tended to use a few theoretical lenses, such as stakeholder theory and institutional-based view, which implies that others are considerably neglected in the empirical investigations. Moreover, the authors also pointed out that the current studies face massive methodological problems. Many scholars have generally endeavored to improve the sophistication and rigor, but researchers still experience serious research gaps in domains such as diversifications in methodological skills, geographical territory, sampling characteristics, and the point of view observing the phenomenon.

We hope that the wide range of gaps illustrated above are cemented by our special issue, and we want to leave some residual agendas as future research avenues on CSR and sustainability strategies. The guest editors would like to thank all reviewers and all those who contributed their studies to the special issue. The guest editors especially passed our gratitude to Nina Tian, who has substantially supported the special issue as an assistant manager. We believe that this special issue could not have been brought to this stage without their generosity and sincere efforts. Again, we wish to give our many thanks to all of them.

Author Contributions: Explanations on special issue background is written by B.I.P., and ten papers chosen by the speical issue are summarized by S.X. All authors have read and agreed to the published version of the manuscript.

Funding: This research received no external funding.

Institutional Review Board Statement: Ethical review and approval are not applicable in that it is an editorail not involving humans or animals.

Informed Consent Statement: Not applicable.

Data Availability Statement: This statement does not necessary for an editorial.

Conflicts of Interest: The authors declare no conflict of interest.

\section{References}

1. Cateora, P.R.; Graham, J.L. International Marketing; McGraw-Hill: New York, NY, USA, 1999.

2. Park, B.I.; Chidlow, A.; Choi, J. Corporate social responsibility: Stakeholders influence on MNEs' activities. Int. Bus. Rev. 2014, 23, 966-980. [CrossRef]

3. Choi, D.; Choi, P.M.S.; Choi, J.H.; Chung, C.Y. Corporate Governance and Corporate Social Responsibility: Evidence from the Role of the Largest Institutional Blockholders in the Korean Market. Sustainability 2020, 12, 1680. [CrossRef]

4. Polonsky, M.; Jevons, C. Global branding and strategic CSR: An overview of three types of complexity. Int. Mark. Rev. 2009, 26, 327-347. [CrossRef]

5. Park, B.I.; Ghauri, P.N. Determinants influencing CSR practices in small and medium sized MNE subsidiaries: A stakeholder perspective. J. World Bus. 2015, 50, 192-204. [CrossRef] 